\title{
The Meaning of an Infinitely Great Velocity
}

\author{
Qing Li \\ Shijiazhuang Traditional Chinese Medical Hospital, Shijiazhuang, China \\ Email: liqingliyang@126.com
}

How to cite this paper: Li, Q. (2021) The Meaning of an Infinitely Great Velocity. Applied Mathematics, 12, 775-792. https://doi.org/10.4236/am.2021.129052

Received: August 3, 2021

Accepted: September 7, 2021

Published: September 10, 2021

Copyright $\odot 2021$ by author(s) and Scientific Research Publishing Inc. This work is licensed under the Creative Commons Attribution International License (CC BY 4.0).

http://creativecommons.org/licenses/by/4.0/

\begin{abstract}
An instantaneous velocity where a moment of the clock only corresponds to an arbitrary distance or position in space cannot be implied in Axiom 1, but it indicates that there is only one dimensional existence, space or time, where a certain moment only corresponds to itself specifically, not to any other time or any given length of space. Further, a definition of velocity that consists of two dimensions representing the relationship between space and time is not valid and there is only one-dimensional space or time that is independent of each other in Axiom 1. As a result, the principle of relativity and the principle of the constant velocity of light are replaced by the principle of an inertial system and the principle of universal invariant velocity in Axiom 1. Unlike two dimensions whose magnitude is determined by the ratio, the magnitude of a single dimension is determined by the unit values of one dimension, which indicates that an infinitely great velocity is meaningless. Further, if the two inertial systems are infinite versus finite in Axiom 3, then this extension of the infinitely great velocity can be defined as inextensible.
\end{abstract}

\section{Keywords}

Infinitely Great Velocity, Universal Invariant Velocity, One-Dimension, The Unit Values of One Dimension

\section{Introduction}

A century ago, Newton and Galileo's absolute view of time and space was replaced by Einstein's special relativity, in which the Galileo transformation formula was substituted by the Lorentz transformation formula. Special relativity based on the principle of relativity and the principle of the constant velocity of light and space-time transformation between inertial system observers are characterized by the observer-independent velocity scale $c$ (i.e., the velocity of light). Twenty years ago, a modified theory of special relativity was postulated by Amelino-Camelia as doubly special relativity [1] (also referred to as deformed special 
relativity), which is based on quantum-gravity arguments. Doubly special relativity, the new relativistic theory in which the space-time transformations between inertial system observers are characterized by two observer-independent scales (in addition to the light velocity scale, there is a second new observer-independent length/momentum scale, the Planck length/momentum). Further, doubly special relativity predicts that a value of $E_{p} \approx 10^{28} \mathrm{eV}$ can be regarded as the maximum value of energy and momentum for fundamental particles, while length/momentum remains unchanged in the space-time inertial frame under the Planck scale.

In 2004, an extension of doubly special relativity was inrtoduced by J. Kowalski-Glikman and Lee Smolin [2] [3], called as triply special relativity, in which there is a third invariant parameter, the cosmological constant $\Lambda$, and so there are now three observer independent scales, $c$ (light velocity), $l_{p}$ (Planck length) and $R=\Lambda^{-1 / 2}$. A extension of the Poincare algerbra [4] [5] can be defined here. For $R \rightarrow 0$, this new algebra reduces to the $\kappa$-Poincare algebra $\left(\kappa=l_{p}^{-1}\right)$, For $l_{p} \rightarrow 0$ it reduces to the de Sitter algebras [6]. The particle motion that follows this new algebra can be taken into account the Poisson structure of the phase space of a relativistic particle.

In mathematics, the mathematical basis for further analysis of relativity has been provided by new mathematical models, such as boundary value problems [7] [8] and discrete mathematics [9] [10] (temporal and spatial discontinuity).

There are two concepts that are logically debatable in relativity. Firstly, the velocity of light is a finite speed, but it is also a limited speed, which indicates that there are infinitely many different speed values to choose from between the velocity of light and a velocity of 0 . This concept can be seen in the Lorentz space-time transformation formula:

$$
X_{1}^{\prime}=X_{1}-v l /\left\{1-(v / c)^{2}\right\}^{1 / 2} \text { and } l^{\prime}=l-v X_{1} /\left\{1-(v / c)^{2}\right\}^{1 / 2}
$$

Secondly, the physical quantities remain unchanged in different inertial systems; in other words, if we do not assume the two inertial systems with different motion states, then the two inertial systems cannot be distinguished. This is called the principle of relativity.

In this paper, based on Axioms 1 [11] and 3 [12], four perspectives of inertial systems that differ from Einstein's special relativity are proposed: 1) the principle of relativity can be replaced by a concept in which an inertial system is only a specific quantity, 2) relative velocity is meaningless; any velocity is constant with respect to any other velocity, 3) a definition of velocity that includes two dimensions (space/time) is not valid and there is only one-dimensional space or time, and 4) unlike two dimensions, where the magnitude is determined by the ratio, with one dimension, it is determined by unit values.

\section{There Is No Instantaneous or Two-Dimensional Velocity}

The relationship between space and time can be expressed in terms of velocity: $V$ 
$=s / t$, where $v$ is the velocity, $s$ is the length of space, and $t$ is time. There are two implications regarding the relationship between space and time arising from this formula: 1) $s$ and $t$ are dimensions that can be compared, and 2) $s$ and $t$ are equivalent. For example, for two velocities of $3 \mathrm{~m} / \mathrm{s}$ and $4 \mathrm{~m} / \mathrm{s}, 1$ second should be equivalent to 3 meters for the first velocity, while 1 second should be equivalent to 4 meters in the second. From Axiom 1, it is known that each length value is specific (because the unit value differs, each length value can only be itself), thus the unit second of time is also specific. If 1 second is equivalent to 3 meters, then 1 second is not equivalent to 4 meters, so the definition of velocity is meaningless in Axiom 1. Another example is that 1 second is a finite number in Axiom 1, so an infinitely great velocity (one second goes into the infinite distance) is not valid in Axiom 1, because 1 second cannot be equivalent to an infinite length. Therefore, in Axiom 1, because the conditions for the two-layered meaning of $v=s / t$ cannot be satisfied simultaneously (that is, the size can be compared and the equivalence can be met simultaneously), a definition of velocity based on two dimensions representing the relationship between space and time cannot be established.

If the two-dimensional property of permissible velocity is true, then certain conditions must be met. As can be seen in Figure 1, the properties that can be compared are eliminated. For a velocity composed of two dimensions, time and space are reduced to dimensions that cannot be compared, that is, the finite and infinite quantities cannot be distinguished, nor can the sizes be compared with each other. For example, 1 meter or 1 second in a general sense can represent any quantity. For the convenience of the following description, this concept is defined as two-dimensions-without-size Axiom 1. Two-dimensions-without-size Axiom 1 is a paradox and meaningless, so the conclusion can be drawn that, in Axiom 1, only one dimension exists and space and time are independent of each other. It is now important to look at some basic properties of Axiom 1.

Property 1 There is only one dimension, space or time, and they are independent of each other. For example, for an event moving at an infinite distance of 1 second, 1 second is a finite quantity, and space at an infinite distance is an infinite quantity. The two quantities are neither equivalent nor dependent on each other. In any other velocity-describing event, the magnitude of space or time is neither equivalent (except for each magnitude itself) nor correlated.

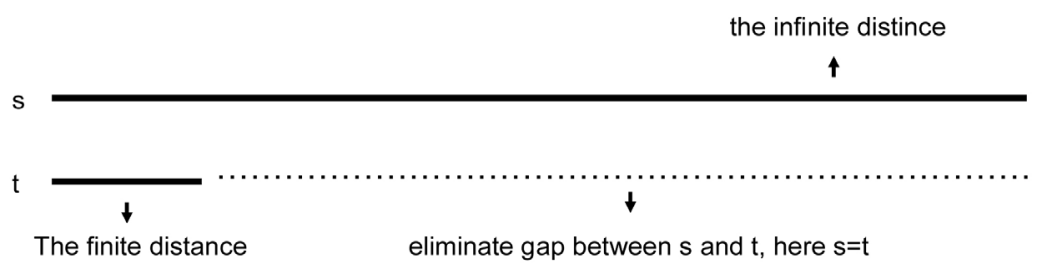

Figure 1. Ability to compare sizes is eliminated and space and time turn into one dimension that cannot be compared in size for a velocity consisting of two dimensions. Further, the finite and infinite quantities cannot be differentiated and sizes cannot be compared with each other. This property is achieved by $s=t$., where $s$ is space and $t$ is time. For convenience, this concept is referred to as two-dimensions-without-size Axiom 1. 
Property 2 There is no instantaneous velocity at infinity. Instantaneous velocity is defined as moving to any point of length in space without time, that is, point 0 corresponds to any point of length in space, a moment of 1 second corresponds to any point of length in space, a certain distance in space (for example, 1 meter) corresponds to any moment in time, and so on.

In Axiom 1, the absence of instantaneous velocity has two implications. First, as mentioned earlier, a single dimension means that there is no velocity with two dimensions that can be compared. Second, the independent existence of space and time does not mean that a certain moment of the clock only corresponds to any distance or position in space; rather, it means that there is only one dimension, space or time. Each value of space corresponds only to itself, not to other quantities, and each value of time corresponds only to itself, not to other quantities; thus, space or time are independent of each other. For example, point 0 only corresponds to point 0 and does not correspond to other quantities (including infinite quantities), while 1 meter only corresponds to 1 meter and does not correspond to other quantities. Unlike the concept of simultaneity/non-simultaneity in relativity, this independence is given a new definition.

The independence of the relationship between space and time can also be illustrated as follows. If we talk about space, it makes no sense for us to talk about time, and if we talk about time, it makes no sense for us to talk about space. A given interval of time does not correspond to any length of space, and a given distance of space does not correspond to any interval of time. Thus, it can be said that, for two different locations in space, whether they exhibit simultaneity or non-simultaneity in time is of no significance; similarly, for two different intervals in time, whether they are in the same or different locations in space is also of no significance.

The absence of instantaneous velocity does not mean that infinite space and infinite time do not exist, just that they exist independently. The absence of instantaneous velocity does not mean that infinite velocity does not exist, nor does it mean that there is only a finite velocity, such as the velocity of light. In Axiom 1 , the velocity of light is only a finite speed (300,000 kilometers and 1 second are both finite), thus it is neither an infinite velocity nor a limit velocity. In Axiom 1, the single dimension dictates that each value corresponds to itself and does not correspond to other values, explaining why a clock at some point in the theory of relativity only corresponds to a certain space with an equal distance or the position itself, not to the concept of the distance or the position of others. However, unlike the description of the theory of relativity, the concept of a single dimension described does not deny that infinite values exist, and there is also no concept of time shortening or space lengthening here. Details on this will be described in Section 6.

\section{Principle of Special Relativity and the Principle of the Constant Velocity of Light}

Concrete descriptions of the single-dimensional properties of Axiom 1 are pro- 
vided in this section. By comparing the concept of time and space in Einstein's special relativity, the properties of a single dimension can be more clearly understood.

Principle of relativity Here, inertial frames and relativity principles are discussed. If $K$ is defined as a Cartesian frame-of-reference system (i.e., an inertial frame), then another Cartesian frame of reference $K$, which is moving uniformly in a straight line with respect to $K$, is also an inertial system (A rotating Cartesian inertial frame is classified as a non-inertial frame and is beyond the scope of this paper). There are three implications here. First, for any coordinate system $K$, all space-time quantities (i.e., spatiotemporal variables) can be expressed in this coordinate system, and all quantities are static relative to $K$. For example, consider two velocity events $s=c t$ or $s=v t$, both of which can be expressed in $K$, where $c$ is the velocity of light and $v$ is any velocity. If $K$ and $K^{\prime}$ without comparison, then the spatiotemporal variables relative to $K$ at rest cannot be used to distinguish the motion state from the spatiotemporal variables relative to $K^{\prime}$ at rest. This is known as the relativity principle. Second, the coordinate system itself and the quantity expressed in the coordinate system can be described by different quantitative terms, such as $K^{\prime}$ moving with velocity $V_{l}$. Any number of values that differ from $V_{l}$ can be described along the $x, y$, and $z$ axes of the coordinate system, such as $s_{1}=c t_{1}$ or $s_{2}=v_{2} t_{2}$, where $c$ is the velocity of light and $v_{2}$ is any velocity. Third, in static coordinate system $K$ with a velocity of 0 , the velocity at all points is 0 . In coordinate system $K^{\prime}$ with a uniform velocity of $V$, the velocity at all points is $v$. The difference between $K^{\prime}$ and $K$ is quantitative, that is, the difference between $v$ and 0 . These concepts apply to Axiom 2 [6].

Principle of the constant velocity of light It has been proven by Michelson's experiment that the speed of light remains constant in Cartesian coordinates with uniform linear motion at any velocity. A moment of a clock corresponds only to a certain distance or position in space equal to itself and does not correspond to any other distances or positions. For example, 1 second only corresponds to 300,000 kilometers (i.e., 1 second is equivalent to 300,000 kilometers) and does not correspond to other distances.

The implications of the transformation of Cartesian coordinates based on these two principles are as follows:

1) In a Cartesian coordinate system that allows instantaneous velocity, relative velocity is meaningful, which indicates that the quantity of velocity for given Cartesian coordinates will vary for Cartesian coordinates with different velocities; that is, the quantity of a given velocity depends on the motion velocity of the Cartesian coordinates. Because a certain moment of a clock corresponds to an arbitrary distance in space, and a certain distance in space corresponds to an arbitrary time of the clock, the transformation between the two Cartesian coordinate systems $K^{\prime}$ and $K$ is arbitrary. In fact, this concept is two-dimensionswithout-size Axiom 1.

2) In a Cartesian coordinate system with a constant velocity of light, the ve- 
locity of light is used as the basis for defining space and time (i.e., light time and light space). For optical space coordinate $X_{I}$ in frame $K$ (stationary coordinates with velocity 0 ), the corresponding optical space coordinates in frame $K^{\prime}$ (a coordinate system with velocity $v$ ) is

$$
X_{1}^{\prime}=1 /(1-v / c) X_{1}\left(X_{1}^{\prime}>X_{1}\right)
$$

This formula is the revised version of the Lorentz transformation. Unlike the relativistic principle, which holds the coordinates of $K^{\prime}$ and $K$ to be identical, here the coordinates for $K^{\prime}$ and $K$ differ due to the fact that all quantities within frame $K$ are stationary with respect to frame $K$, but they are not stationary with respect to frame $K^{\prime}$.

3) The formula $X_{1}^{\prime}=X_{1}-c t$ cannot be established for a comparison of the coordinates between the two frames $K^{\prime}$ and $K$ because relative velocity is nonexistent in relativity of revised version; in other words, a minus sign in the formula does not exist.

4) It is known from (2) that the same proportional extension of $K^{\prime}$ and $K$ coordinates for the two coordinate systems is carried out as

$$
X_{1}^{\prime}: 1 /(1-v / c) X_{1} \text {, }
$$

where $X_{1}^{\prime}=1 /(1-v / c) X_{1}$.

The purpose of this formula is to facilitate a comparison of the coordinate transformation of the two coordinate systems so that the two coordinates are compared at the same length value and the same scale of time.

5) According to (2), $X_{1}^{\prime}=1 /\left\{1-(v / c)^{2}\right\}^{1 / 2}\left(X_{1}-c t\right)$ [13]

The Lorentz transformation is meaningless; instead, $X_{1}^{\prime}$ is given by the formula

$$
X_{1}^{\prime}=1 /(1-v / c) X_{1} .
$$

Therefore, the notion that frames $K^{\prime}$ and $K$ coincide at origin 0 is meaningless and frame $K^{\prime}$ does not start at origin 0 .

6) From (2), because the $K^{\prime}$ and $K$ coordinates are different, the two Lorentz transformation equations

$$
X_{1}^{\prime}=1 /\left\{1-(v / c)^{2}\right\}^{1 / 2}\left(X_{1}-c t\right)
$$

and

$$
X_{1}^{\prime}=1 /\left\{1-(v / c)^{2}\right\}^{1 / 2}\left(X_{1}-c t^{\prime}\right)
$$

are not valid, and they are replaced by the following two equations:

$$
X_{1}^{\prime}=c t_{1}^{\prime} \text { and } X_{1}=c t_{1}
$$

Here $X_{1}^{\prime}=1 /(1-v / c) X_{1}$ and $t_{1}^{\prime}=1 /(1-v / c) t_{1}$.

The main characteristics of these two equations that differ from the Lorentz transformation are that their coordinates are given by $X_{2}^{\prime}-X_{1}^{\prime}>X_{2}-X_{1}$ (Figure 2). 
1

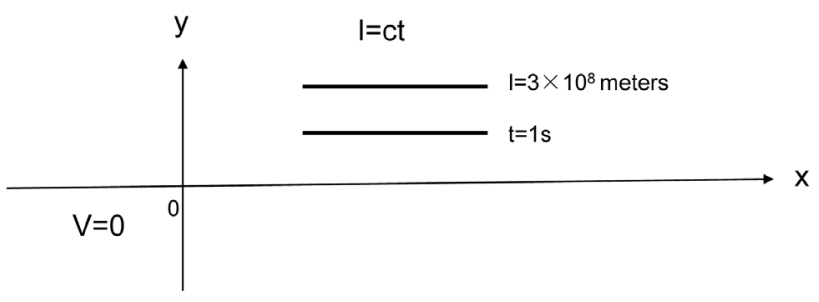

2

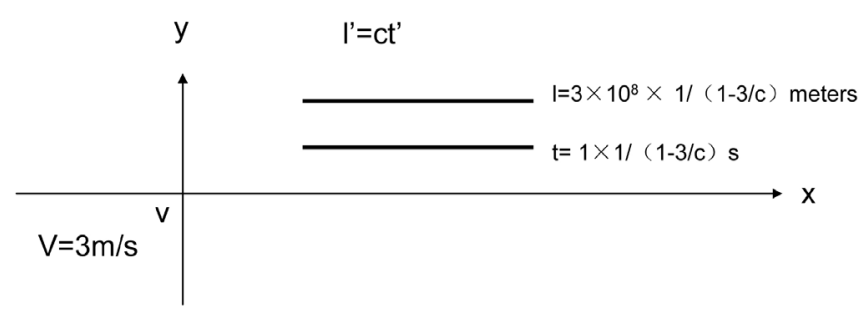

Figure 2. (1) With stationary Cartesian coordinates, $3 \times 10^{8}$ meters is equivalent to 1 second, and the time beat is given as 1 second $\left(=3 \times 10^{8}\right.$ meters $)$. (2) For Cartesian coordinates with a velocity of $3 \mathrm{~m} / \mathrm{s}, 3 \times 10^{8} \times 1 /(1-3 / c)$ meters is equivalent to $1 \times 1 /(1-$ $3 / c)$ seconds, with the time beat given as $1 /(1-3 / c)$ seconds $\left(=3 \times 10^{8} \times 1 /(1-3 / c)\right.$ meters). It is concluded from relativity that a velocity of $3 \mathrm{~m} / \mathrm{s}$ will be given in the form of 3 $\times 10^{8} \times 1 /(1-3 / c)$ meters $/ 1 \times 1 /(1-3 / c)$ seconds.

\section{Principle of an Inertial System and the Principle of Universal Invariant Velocity for Axiom 1}

Principle of an inertial system for Axiom 1 It is known from Axiom 1 that relativity is not true. As shown in Figure 3, in Axiom 1, each inertial system is described by a unit value, such as 2,3 , or 4 . In this system, the properties of the proportional extension of two inertial frames can then be considered. For example, two inertial frames 2:1 are compared, with the following extension ratios being 4:2, 6:3, 8:4, etc. In this comparison of inertial frames, we also consider a unit extension of $4: 2$ to the same ratio, meaning the next extension ratio is $8: 4$, followed by $12: 6$, and so on. Comparing $8: 4$ to the same scale unit extension, the next is $16: 8$, followed by $24: 16$, and so on. Although the ratio is $2 / 1$, the two units extend differently and cannot replace or offset each other because of the different units (the former is in units of 4 and the latter is in units of 8). Therefore, relative velocity is meaningless in Axiom 1, which means that a given quantity, as distinct from the other units of quantity, can only be itself and not any other quantity; this particular quantity thus represents only one state, not any other state, and the Cartesian coordinate system does not apply in Axiom 1. As a result, the properties of the inertial system of relativity need to be revised.

First, in the principle of an inertial system for Axiom 1, an inertial system is a specific quantity and only represents a state, so the motion of all quantities is absolute, and any comparison of the motion of two quantities is also absolute.

Second, the absoluteness of this motion negates the relativity principle of relativity theory. Thus, it can be said that a stationary concept is also meaningless in 


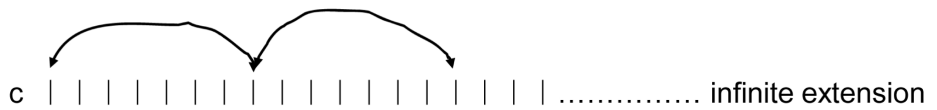

Figure 3. In Axiom 1, each inertial frame can be described by unit quantities, such as 1, 2, 4, etc. Although the ratio is $2: 1$, the units are different ( $b$ is in 4 units, and $c$ is in 8 units), so the proportional extension of the units differs and cannot replace or offset each other.

the principle of an inertial system for Axiom 1. In the principle of relativity, if $K$ is a stationary Cartesian inertial system (i.e., the coordinates for all space and time variables are stationary relative to $K$ ), $K^{\prime}$ is a moving Cartesian inertial system relative to $K$ with velocity $v$ so, following the Axiom 1 inertial system principle, it is meaningless to talk about all space-time variables as stationary relative to $K$, and $K$ does not exist as an inertial system at rest. It can thus be said that the Cartesian coordinate system cannot describe the distribution of quantities in space and time and that all-embracing variables in space and time that stand stationary relative to the coordinate system do not exist.

Thirdly, unlike the coordinate system, which must be described with two different terms as discussed above, the inertial system in Axiom 1 only has a quantitative term description, such as an inertial system whose space units are 1, 4, 8, or $\mathrm{N}$ (all are multiples of 0 ). An inertial frame represents only a specific quantity, that is, only a state.

Fourth, consider the comparison of two inertial systems in Axiom 1, such as the 4:1 proportionate extension, where inertial system 4 extends in units of 4 , and inertial system 1 extends in units of 1 . The extension of the two inertial systems involves an infinite number of different comparisons except that the extension ratio of 4:1 is fixed. For example, the extension of inertial system 4 is 4,8 , 12,16 , etc. (infinitely many different quantities), while the extension of inertial system 1 is $1,2,3$, 4, etc. (infinitely many different quantities). Thus, the difference between the two inertial systems is not a difference of one quantity, but an infinite number of quantities.

The Cartesian coordinate system which thus describes the difference in a quantity cannot be applied to describe infinitely many differences. In addition, in a Cartesian coordinate system moving with velocity $\mathrm{v}$, all of the points moving with velocity $v$ are meaningless because, in Axiom 1, point 0 only represents point 0 and cannot be endowed with other concepts, such as point 0 moving with a velocity that is two-dimensional.

Principle of universal invariant velocity for Axiom 1 In Axiom 1, each quantity is a specific quantity, that is, it is itself rather than any other quantity. Thus, 
we reasonably conclude that, in Axiom 1, any velocity is itself, not any other velocity (where the essence of the concept of velocity is single-dimensional space or time). Each velocity is thus constant relative to other velocities, not just the velocity of light. This property is defined as the principle of universal velocity invariance for Axiom 1.

\section{Velocity Is One-Dimensional}

Common misconceptions about velocity can be cleared up using these two principles.

1) Velocity is two-dimensional, and there is an instantaneous velocity going to the infinite distance. Here space and time are independent of each other, that is, a certain moment of the clock corresponds to any distance or position in space. For example, 1 second corresponds to any length, which is the Newtonian absolute space-time view. Because a moment of a clock corresponds to any distance or position in space, this means that velocity is variable, that is, the size of one velocity depends on how much it corresponds to other velocity, and velocity can be added or reduced. Therefore, the concept of a specific velocity does not exist here. The single-dimensional properties and universal-velocity-invariant properties of Axiom 1 deny the correctness of this concept. This property is essentially two-dimensions-without-size Axiom 1, which means that a Galilean transformation is meaningless.

2) Velocity is two-dimensional and can be compared in size, in which there is no instantaneous velocity extending to infinity, and the velocity of light is a finite magnitude of velocity that is also a limit velocity. Here, the principle of relativity can be applied. Because the invariability of the velocity of light (i.e., it remains constant for Cartesian coordinates at any velocity) has been experimentally confirmed, the velocity of light has a privileged position as the basis for defining space and time (i.e., light time and light space), which is known as relativistic space-time. Here, one second of the clock corresponds to a space length of only 300,000 kilometers (i.e., 1 second is equivalent to 300,000 kilometers), two seconds corresponds to $600,000 \mathrm{~km}$ (i.e., 2 seconds is equivalent to $600,000 \mathrm{ki}$ lometers), and so on. Here, 1 second does not correspond to other distances, such as 3 meters. Therefore, the notion of a velocity of $3 \mathrm{~m} / \mathrm{s}$ (i.e., 1 second is equivalent to 3 meters) makes no sense with relativity. Speed events of $3 \mathrm{~m} / \mathrm{s}$ are given in light time and in light space (Figure 2). As a result, the space-time properties of two inertial systems $K^{\prime}$ and $K$ (for example, an inertial system with a velocity of $3 \mathrm{~m} / \mathrm{s}$ is compared with an inertial system with a velocity of 0 ) have the following characteristics. From the observation of $K^{\prime}$, the time of $K$ is prolonged, and the space is shortened. As observed from $K$, the time of $K^{\prime}$ is shortened and the space is elongated (Figure 4). The single-dimensional nature of Axiom 1 and the infinity of space-time deny the correctness of this concept.

3) Velocity is two-dimensional, and there is an infinite but not instantaneous velocity. The inertial system principle and universal velocity invariant principle 


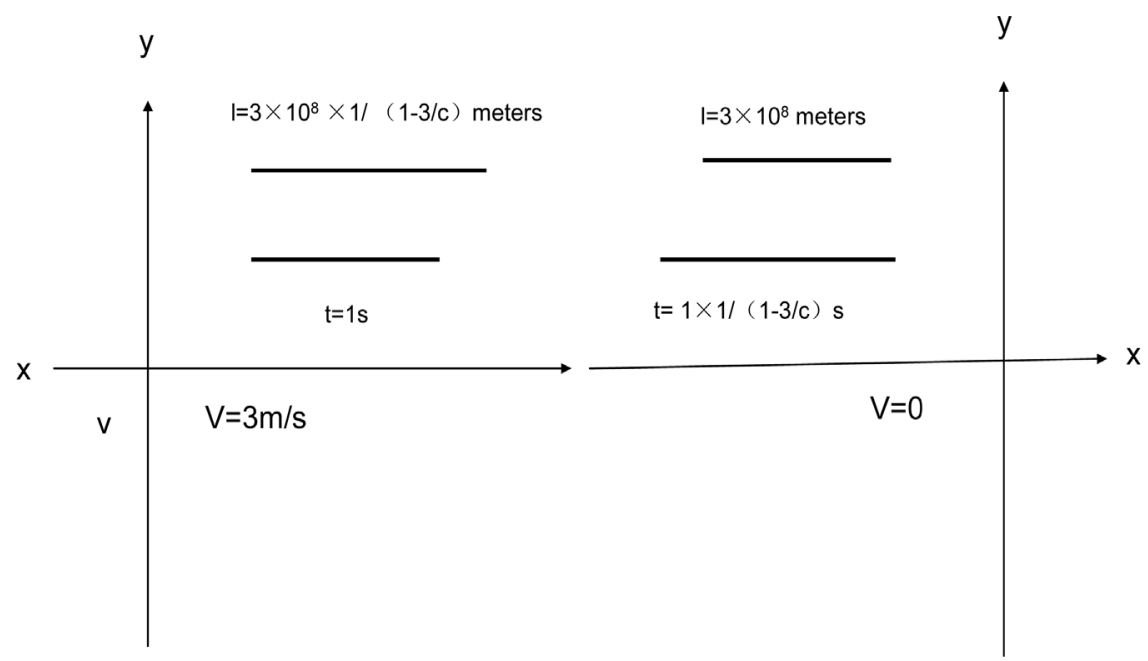

Figure 4. Space-time properties for a comparison of two inertial systems $K^{\prime}$ and $K$. For example, when inertial system $K^{\prime}$ with a velocity of $3 \mathrm{~m} / \mathrm{s}$ is compared with the inertial system $K^{\prime}$ with a velocity of 0 , when observed from $K$, the time of $K$ is lengthened and space is shortened. In contrast, when observed from $K$, the time of $K^{\prime}$ is shortened and space is elongated.

follow Axiom 1 and do not follow the relativity principle; in other words, a certain moment of the clock only corresponds to a specific distance in space and does not correspond to other distances. For example, infinite time only corresponds to infinite distance, not to a finite distance (such as a distance of 1 meter), and a finite clock scale only corresponds to a finite distance, not to infinite distance. Unlike (2), here the velocity of light is not the only basis for defining space and time, allowing for the existence of arbitrary values for velocity. Two implications arise from this arbitrary velocity, First, it is meaningful that the space-time is not equivalent. For example, although 1 second is equivalent to 300,000 kilometers, it is not equivalent to 3 meters at $3 \mathrm{~m} / \mathrm{s}$, but a velocity of $3 \mathrm{~m} / \mathrm{s}$ is meaningful. Second, the magnitude of velocities can be compared. For example, the velocity of light has the same quantitative value as the unit of time for $3 \mathrm{~m} / \mathrm{s}$. The stationary state of it, unlike the Cartesian coordinates of relativity, should be given as $0 / \infty$. The single-dimensional nature of Axiom 1 denies the correctness of this concept. The essence of (2) and (3) are still Axiom 2.

4) There is only one-dimensional space or time, and there is no concept of velocity, regardless of whether it is infinite or finite. Space and time are independent of each other here, with a certain moment of the clock only corresponding to the moment itself, not to other moments or any distance or position in space. Likewise, a certain distance in space corresponds only to its own distance, not to any other distance in space or any time of the clock. Therefore, the inertial system principle of Axiom 1 and the universal velocity invariant principle are followed here. Velocity has become single-dimensional space or time and Only the finite and infinite space, or finite and infinite time can be talked about. If the concept of velocity is being referred to, the two values (distance in 
space and time in time) are neither equivalent nor dependent on each other. The essence of (4) is Axioms 1 and 3. For instance, for a velocity event moving to infinite distance in 1 second, it can be seen from the above definition that 1 second is not equivalent to infinite distance, because the concept of a single event of infinite speed being associated with time and space is meaningless. Rather, 1 second and infinity exist independently as two events: an event of infinitely great space in one dimension and another event of 1 second in time in one dimension.

\section{Meaning of One-Dimensional Velocity}

By comparing (3) and (4), we can outline their specific features. For feature (3), the velocity is determined by the ratio of the two dimensions. There is an infinitely great velocity, expressed by $\infty / \mathrm{dl}$, where $\infty$ is infinitely great and $\mathrm{dl}$ is infinitesimally small. A state of zero velocity is denoted as $\mathrm{dl} / \infty$, and $\mathrm{dl}$ does not equal 0 here (due to the nature of Axiom 2). Feature (3) follows the inertial system principle of Axiom 1 and the universal velocity invariant principle, but does not follow the relativity principle, so the Cartesian coordinate system does not apply to (3). For example, a Cartesian coordinate system with a velocity of 0 (i.e., static) does not exist. Motion is absolute and there is no static state, so a comparison of two inertial frames is a comparison of two specific states. For example, let the inertial system $K^{\prime}$ be the infinite velocity and the inertial system $K$ velocity be 0 . Figure 5 shows a comparison of the two inertial systems. Their spatiotemporal properties are determined by two points ( $a$ and $b$ ). When observed from $K^{\prime}$, the time lengthens and space shortens in $K$. In contrast, from the point of view of $K$, the time in $K^{\prime}$ is shortened and the space is lengthened. Because Cartesian coordinates do not apply to (3), the Lorentz transformation does not make sense here. The transformation of the magnitude of space-time is a universal transformation, which is determined by the magnitude of $a$ and $b$.

Axiom 1 is different from the relativistic principle about the concept of inertial system. In Relativity, A set of inertial systems is a set of Cartesian coordinates, for any given coordinate system $K^{\prime}$, all space-time quantities (i.e., spatiotemporal variables, all states) can be expressed in this coordinate system, as long as they are stationary in this inertial frame. For instance, For an inertial

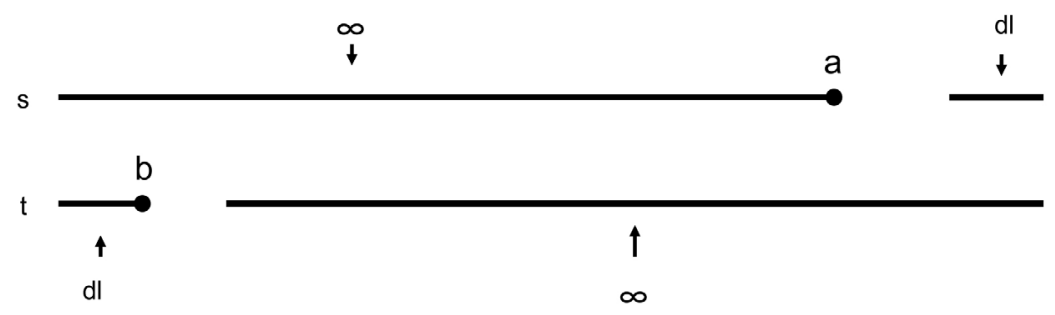

Figure 5. Comparison between two inertial systems in (3). For example, the inertial system $K^{\prime}$ has an infinitely great velocity, and inertial system $K$ has a velocity of 0 (i.e., a stationary state). Their space-time properties are determined by two points ( $a$ and $b$ ). Observed from $K^{\prime}$, the time in $K$ is lengthened $(\infty)$ and space is shortened (dl), When observed from $K$, the time in $K^{\prime}$ is shortened (dl) and space is elongated $(\infty)$. 
frame $K$ with a speed of 0 , the speed of light can be expressed in this inertial frame. Therefore, in the theory of relativity, an inertial frame $K^{\prime}$, which moves at the velocity $\mathrm{v}$ relative to the stationary inertial frame $K$, will have a longer space and a shorter time, where the velocity of light becomes the minimum value of the space-time ratio relation. However, in the principle of an inertial system for (3) or Axiom 1, an inertial system is a specific quantity and only represents a state, Where a Cartesian coordinate system is no longer applicable, and its minimum value of the space-time ratio relation is $\mathrm{dl} / \infty$ in (3) and is 0 in Axiom 1 , That is to say, because of axiom 1, the concept of Cartesian inertial frame and the relativistic principle in Relativity becomes meaningless.

Feature (4) can now be considered. The single-dimensional nature means that its space-time nature is determined by one point, not two (a and b). Therefore, instead of two dimensions being determined by the ratio, the size of a single dimension is determined by a one-dimensional unit value (which varies by unit number). Therefore, it is meaningless to lengthen or shorten the space-time of two inertial systems in a two-dimensional state.

How the extension of velocity representing two dimensions differs from the extension of space or time representing one dimension can now be discussed. It is suggested by Axiom 2 that the space-time extension of a velocity can reach infinite distance and that the ratio of the velocity is arbitrary and either finite or infinite. As shown in Figure 6, in a comparison of two Cartesian inertial systems moving at different velocities, their space-time extension can also reach infinite distance. In Axiom 1, quantitative values extend in units of 0 (i.e., $10,20,30$,

extension to infinte distance

a

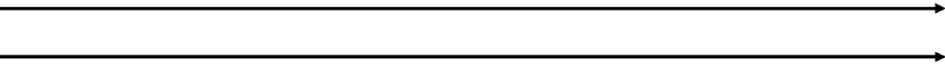

b
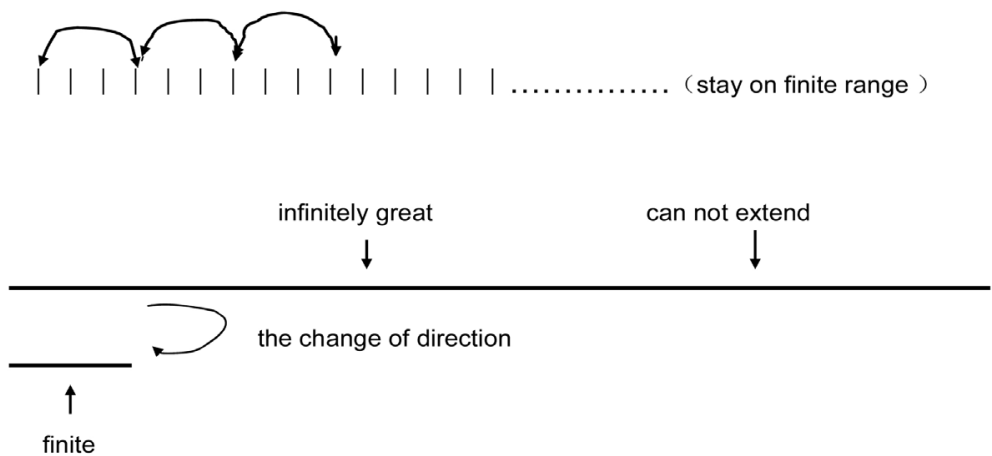

Figure 6. (a) It is suggested by Axiom 2 that the space-time extension of a velocity can reach infinite distance, the ratio of the velocity is arbitrary and either finite or infinite. (b) It is known from Axiom 3 that if two inertial systems are compared for finite quantities, then the extension of the quantities of the two inertial systems must remain within the finite range and do not reach infinite distance. (c) If two inertial systems are infinite versus finite, then it is known from Axiom 3 that a change of direction means it is infinitely great and the finite is not part of infinitely great, so this extension of infinitely great is defined to be inextensible. 
and so on). The extension of two different values (i.e., two inertial frames) is carried out using an arbitrary integer () 1 that is a multiple of 0 and is carried out in units. The minimum magnitude is one 0 . Unlike (3), where there is an inertial system with an infinite approach velocity of $0(\mathrm{dl} / \infty)$, the nearest 0 inertial system in Axiom 1 is two 0 inertial systems. In Axiom 1, the uniqueness of infinity determines that the formula $1 / 0=\infty / 1=\infty$ is not true and that only the formula that $\infty / 0=\infty$ is true, meaning the formula $300,000 \mathrm{~km} / 0=\infty / 300,000 \mathrm{~km}=$ $\infty$ is not true. For each finite length (for example, 1 meter) there is a finity, not an infinity, so 300,000 kilometers is not sufficient to carry an infinite amount of burden. The existence of Axiom 1 means that there is only a finite number of quantities to choose from between 0 and 1 second or between 0 and 300,000 kilometers, and there must be an infinite number of quantities to choose from between 0 and $\infty$ (units of seconds or kilometers). Therefore, the velocity of light is not an ultimate velocity, and putting the velocity of light into a special superior position lacks any profound basis in physics.

In Axiom 1, quantitative values extend in units of 0 (i.e., $10,20,30$, and so on). The extension of two different values (i.e., two inertial frames) is carried out using an arbitrary integer () 1 that is a multiple of 0 and is carried out in units. This implies that it is meaningless to compare any two inertial systems of non-integral numbers, such as the inertial system with $4 \mathrm{~m} / \mathrm{s}$ versus the inertial systems with $3 \mathrm{~m} / \mathrm{s}$, and that the extension of the two in the same proportion is also meaningless. So the Lorentz space-time transformation formula is replaced here by the space-time transformation of an arbitrary integer () 1(notice here that it maxmium value is $\infty / 0$, not $1 / 0$ ).

Because Axiom 3 is a modification of Axiom 1 (that is, Axiom 3 retains some of the properties of Axiom 1), if two inertial systems involve a comparison of finite values, then these two inertial systems extend only in a finite range and cannot extend to infinity (derived from Axiom 3). If the two inertial systems are unlimited (i.e., infinite) compared with limited amount of, so learn from Axiom 3 , in which the direction of change means infinitely great, and the finity is not part of infinitely great, that then for infinity (infinitely great) has two meanings. Firstly, it is the largest unit (with an infinitely great unit), i.e., there is no bigger or smaller amount, and therefore this extension of infinitely great is defined as inextensible (Figure 6). Secondly, the change in direction means that it cannot be added, subtracted, multiplied or divided, and that it is not a finite component, so it does not vary with the corresponding value of a finite number. Therefore, the Lorentz transformation in the two inertial systems of relativity and the modified Lorentz transformation (corresponding to changes in time and space length), or other magnitude and value transformations (which apply to Axioms 1 and 2), are meaningless in Axiom 3. Instead of the spatiotemporal coordinate transformation or numerical transformation of the two inertial systems defined in Axioms 1 and 2 (only in the motion of uniform linear velocity), the spatiotemporal transformation of the two different inertial systems in Axiom 3 only changes in one direction, which is a unique quantity-value transformation and represents 
all quantity-value transformations (not only in the motion of uniform linear velocity but also in non-uniform linear velocity).

Therefore, instead of two dimensions being determined by the ratio, the size of a single dimension is determined by a one-dimensional unit value. A comparative illustration is seen below. In Axiom 2, The velocity $v$ is expressed by the formula $v=s / t$, If $v$ is a finite value, $s$ and $t$ are values that can extend to infinity. If $v$ is a infinite value, $s$ is values that can extend to infinity and $t$ is finite values can not extend to infinity, or $\mathrm{s}$ is finite values that can not extend to infinity and $t$ is 0 values (infinitesmal) and $s$ is infinite values that can extend to infinity and $t$ is 0 values (infinitesmal) as well. However, In Axiom 3 this is not true, the concept of velocity has been replaced by one dimensional space or time. The numerical size of a one-dimensional space is determined by the unit value. The quantity 2 or 4 , for example, are two different finite unit values, and the largest unit value is infinity. For a finite quantity, an extension in units can only stay in the finite range, and cannot extend to infinity. And for an infinite great number, it doesn't make sense to extend in its units, because it's not extensible, and for an infinite number, you cannot do the operations of addition, subtraction, multiplication or division on it.

The transition from finity to infinity is not a continuous process, but a jumping process. Therefore, there is no intermediate quantity between finity and infinity. The reason for this jumping process is that the infinity is defined as a quantity that can never be reached by extension forever, and the finite accumulation (extension) can only stay in a finite range. Therefore, the transition from finity to infinity must be completed by changing the direction. Consequently, instead of the space-time transformation represented by Lorentz formula, the space-time transformation of axiom 3 is represented by the following formula.

1) Lorentz space-time transformation formula (space coordinates):

$$
\begin{gathered}
X_{1}=n \\
X_{1}^{\prime}=n /\left\{1-(v / c)^{2}\right\}^{1 / 2}
\end{gathered}
$$

2) Space transformation formula of Axiom 3:

$$
\begin{gathered}
X_{1}=n \\
X_{1}^{\prime}=x_{1}^{\prime} / x_{1} \times n
\end{gathered}
$$

Here $x_{1}^{\prime}$ is coordinates of $K^{\prime}$ and $x_{1}$ is coordinates of $K$ corespondingly, $x^{\prime}$ and $x$ must be finite quantities, not infinite quantities. The space quantities given by $X_{1}=n$ in $K$, while for $K^{\prime}$, the space quantities is given by $X_{1}^{\prime}=x_{1}^{\prime} / x_{1} \times n$.

$$
\begin{gathered}
X_{1}=n \\
X_{1}^{\prime}=\infty / 0
\end{gathered}
$$

Here $x_{1}^{\prime}$ is coordinates of $K^{\prime}$ and $x_{1}$ is coordinates of $K$ corespondingly, $x$ must be finite quantities, $x^{\prime}$ must be infinite quantities. The space quantities given by $X_{1}=n$ in $K$, while for $K^{\prime}$, the space quantities is given by $X_{1}^{\prime}=\infty / 0$. Since this infinitely great is defined as space quantities that compresses any quantities 
outside of it to nothing, this extension of infinitely great is also defined as inextensible. So here $X_{1}^{\prime} \quad\left(X^{\prime}=X_{1}^{\prime}\right)$ is a fixed value, which does not increase or decrease with the increase or decrease of the $\mathrm{n}$ value of the formula $X_{1}=n$. In addition, It must be emphasized that $1 / 0$ in Lorentz formula cannot appear in the formula $X_{1}^{\prime}=\infty / 0$, because in axiom 3, the value of light speed (1 second or 300,000 kilometers) cannot exist as an infinite value, so the formula $X^{\prime}=\infty / 0$ is unique.

3) Lorentz space-time transformation formula (time coordinates):

$$
\begin{gathered}
t_{1}=n \\
t_{1}^{\prime}=n /\left\{1-(v / c)^{2}\right\}^{1 / 2}
\end{gathered}
$$

4) Time transformation formula of Axiom 3:

$$
\begin{gathered}
T_{1}=n \\
T_{1}^{\prime}=t_{1}^{\prime} / t_{1} \times n
\end{gathered}
$$

Here $t_{1}^{\prime}$ is coordinates of $K^{\prime}$ and $t_{1}$ is coordinates of $K$ corespondingly, $t^{\prime}$ and $t$ must be finite quantities, not infinite quantities. The time rhythm given by $T_{1}=$ $n$ in $K$, while for $K^{\prime}$, the time rhythm is given by $T_{1}^{\prime}=t_{1}^{\prime} / t_{1} \times n$.

$$
\begin{gathered}
T_{1}=n \\
T_{1}^{\prime}=\infty / 0
\end{gathered}
$$

Here $t_{1}^{\prime}$ is coordinates of $K^{\prime}$ and $t_{1}$ is coordinates of $K$ corespondingly, $t$ must be finite quantities, $t^{\prime}$ must be infinite quantities. The time rhythm given by $T_{1}=$ $n$ in $K$, while for $K^{\prime}$, the time rhythm is given by $T_{1}^{\prime}=\infty / 0$. Since this infinitely great is defined as time rhythm that compresses any quantities outside of it to nothing, this extension of infinitely great is also defined as inextensible. So here $T_{1}^{\prime}\left(T^{\prime}=T_{1}^{\prime}\right)$ is a fixed (unchanged or inextensible) value, which does not increase or decrease correspondingly with the increase or decrease of the $n$ value rhythm ( $n$ value rhythm extensible) of the formula $T_{1}=n$. In addition, It must be emphasized that $1 / 0$ in Lorentz formula cannot appear in the formula $T_{1}^{\prime}=\infty / 0$, because in axiom 3 , the value of light velocity ( 1 second or $300,000 \mathrm{ki}-$ lometers) cannot exist as an infinite value, so the formula $T^{\prime}=\infty / 0$ is unique.

In Axiom 3, an inertial system is a specific quantity and only represents a state, Therefore, when two finite inertial frames $K^{\prime}$ and $K$ are compared, the extension of space length or time rhythm of $K^{\prime}$ and the extension of space length and time rhythm of $K$ are carried out in a fixed proportion $\left(x_{1}^{\prime} / x_{1}\right)$, Which we can call this fixed proportion as invariance. However, when the infinite inertial frames $K^{\prime}$ and finite inertial frames $K$ are compared, because $K^{\prime}$ is inextensible, we can also call this non-extensibility as permanent change in contrast to the invariance of extension of two finite inertial frames. This permanent change can be called as one quantitative continuum of infinite quantities of infinite dimensions indicated by the change of direction. It can be explained here, and if I were given a curve that goes to infinite distance, this curve is a member of all states, and it is interleaved distribution with other curves that belong to all states. Thus 
this all states can be described as the permanent change relative to an finite quantities and cannot be described by a Cartesian coordinate system that base on invariance. Since all the formulas describing the geometric characteristics of space or time are based on invariance, they cannot be used to describe this permanent change. So the Lorentz space-time transformation formula is replaced here by the space-time transformation of this permanent change. This permanent change can be also applicable to non inertial systems, as discussed in my other paper.

\section{Conclusions}

1) It is concluded from Axiom 1 that a definition of velocity in relativity that consists of two dimensions representing the relationship between space and time is not valid and there is only independent one-dimensional space or time in Axiom 1. As a result, the principle of relativity and the principle of the constant velocity of light are substituted by the principle of the inertial system of Axiom 1 and the principle of universal invariant velocity of Axiom 1.

2) Unlike two dimensions whose magnitudes of space and time are determined by the ratio between the two, the magnitude of a single dimension is determined by the unit values of one dimension, which indicates that any velocity (including infinitely great velocity) is meaningless and there is only infinitely great space in one dimension and an infinitely long time in one dimension.

3) Because Axiom 3 is a modification of Axiom 1, it retains some properties of Axiom 1 despite its new properties. Unlike Axiom 1, in which the transition from finite to infinite is a continuous process, in Axiom 3, the transition from finite to infinite involves a leap, thus, if the extensions are within the range of finite quantities for two inertial systems in Axiom 3, they must only stay in the finite range and do not reach infinite distance. If these two inertial systems are infinite versus finite, then it is known from Axiom 3 that the change in direction means infinite great, and this extension of infinite great can be defined as inextensible.

Some readers want to ask, how can axiom 1 be applied to our commonly used physical quantities, such as length meters and time seconds. In axiom 1 , point 0 (singularity) is the minimum value of physical quantity, so some readers will ask, how many such singularities does 1 meter contain? As mentioned earlier, axiom 3 is a modified version of axiom 1 . In axiom 3, such singularity does not exist, but axiom 3 retains all the physical characteristics of axiom 1 . In axiom 3, only the one quantity continuum representing infinity exists, but there is no finite quantities. Therefore, whether 1 meter is finity or infinity has lost its significance. The concept of 1 meter is an approximate comparison concept in axiom 3, that is, we artificially extract a part (called as $A$ ) from this one quantity continuum, and if all other parts (called as $B$ ) have a finite value compared with $\mathrm{A}$ and 1 meter is in the range of $B / A$, so we give the length of 1 meter the meaning of actual physical quantity. Since this paper mainly discusses the inertial system 
characteristics of axiom 1 and axiom 3, other physical characteristics will be discussed in detail in other papers.

There are some limitations to this study presently. First, there have been no direct observations made to confirm the conclusions of this study. Second, due to the difficulty of observing infinity, this research rests only on logical reasoning, but this does not prevent it from redefining or approximating the relationship between the physical quantities in the observable finite range of time and space. In other words, the conclusion is still applicable to physical quantities within this range. Thus, one of the greatest benefits of this study may be that we can redefine the mass-energy equation.

\section{Prospects}

This paper discusses the concept of inertial systems in Axiom 1 (i.e. uniform linear motion), so the reader may ask, how does Axiom 1 define the concept of non-inertial systems (e.g., acceleration or curved motion)? Because two dimensions do not exist in Axiom 1, neither do many dimensions, so how does a single dimension define a non-inertial system (e.g., acceleration)? I will discuss this issue in detail in my next paper [14].

\section{Conflicts of Interest}

The author declares no conflicts of interest regarding the publication of this paper.

\section{References}

[1] Amelino-Camelia, G. (2002) Relativity in Spacetimes with Short-Distance Structure Governed by an Observer-Independent (Planckian) Length Scale. International Journal of Modern Physics D, 11, 35-59. https://doi.org/10.1142/S0218271802001330

[2] Freidel, L., Kowalski-Glikman, J. and Smolin, L. (2004) $2+1$ Gravity and Doubly Special Relativity. Physical Review D, 69, Article ID: 044001.

https://doi.org/10.1103/PhysRevD.69.044001

[3] Kowalski-Glikman, J. and Smolin, L. (2004) Triply Special Relativity. Physical Review $D$, 70, Article ID: 065020. https://doi.org/10.1103/PhysRevD.70.065020

[4] Lukierski, J., Ruegg, H., Nowicki, A. and Tolstoi, V.N. (1991) Q Deformation of Poincare Algebra. Physics Letters B, 264, 331. https://doi.org/10.1016/0370-2693(91)90358-W

[5] Kowalski-Glikman, J. and Nowak, S. (2002) Doubly Special Relativity Theories as Different Bases of Kappa-Poincare Algebra. Physics Letters B, 539, 126. https://doi.org/10.1016/S0370-2693(02)02063-4

[6] Lukierski, J., Nowicki, A. and Ruegg, H. (1991) Real Forms of Complex Quantum Anti-De Sitter Algebra U q(Sp(4:C)) and Their Contraction Schemes. Physics Letters B, 271, 321-328. https://doi.org/10.1016/0370-2693(91)90094-7

[7] Iqbal, M.K., Abbas, M. and Wasim, I. (2018) New Cubic B-Spline Approximation for Solving Third Order Emden-Flower Type Equations. Applied Mathematics and Computation, 331, 319-333. https://doi.org/10.1016/j.amc.2018.03.025

[8] Khalid, N., Abbas, M. and Iqbal, M.K. (2019) Non-Polynomial Quintic Spline for 
Solving Fourth-Order Fractional Boundary Value Problems Involving Product Terms. Applied Mathematics and Computation, 349, 393-407. https://doi.org/10.1016/j.amc.2018.12.066

[9] Khalid, N., Abbas, M., Iqbal, M.K., Singh, J. and Ismail, A.I.M. (2020) A Computational Approach for Solving Time Fractional Differential Equation via Spline Functions. Alexandria Engineering Journal, 59, 3061-3078.

https://doi.org/10.1016/j.aej.2020.06.007

[10] Akram, T., Abbas, M., Riaz, M.B., Ismail, A.I. and Ali, N.M. (2020) An Efficient Numerical Technique for Solving Time Fractional Burgers Equation. Alexandria Engineering Journal, 59, 2201-2220. https://doi.org/10.1016/j.aej.2020.01.048

[11] Li, Q. (2021) A Geometry Consisting of Singularities Containing Only Integers. https://doi.org/10.21203/rs.3.rs-219046/v1

[12] Li, Q. (2021) The Meaning of the Infinitely Great. https://doi.org/10.22541/au.160822935.50569408/v2

[13] Einstein, A. (1979) The Meaning of Relativity. Beijing Science Press, Beijing, 22-23.

[14] Li, Q. (2021) The Meaning of Accelerated Motion. Applied Mathematics, 12, 535-545. https://doi.org/10.4236/am.2021.127037 